\title{
notes
}

\section{Les utilisations non médicales des rayonnements ionisants en Tunisie}

\author{
Bilan actuel, problèmes de radioprotection \\ H.A. GHARBI, J.C. VICEL, M.J. GHEZAIEL, H. CHARNI, S. M'TIMET* \\ (Manuscrit reçu le 5 décembre 1988)
}

La Tunisie ne dispose pas de centrale nucléaire ni de réacteur de recherche. Cependant, l'utilisation des rayonnements ionisants dans les domaines médicaux, industriels, et de recherche tend à se généraliser sur l'ensemble du territoire dans des domaines très variés. Les auteurs ont déjà publié les résultats d'enquêtes concernant l'utilisation médicale des rayonnements ionisants en Tunisie [6]. Cet article présente les résultats d'un ensemble d'enquêtes concernant l'utilisation non médicale des rayonnements ionisants.

La présentation d'un tel travail nous semble justifiée pour les raisons suivantes:

- permettre au médecin radiologue et non radiologue exerçant en Tunisie, d'évaluer le type et l'ampleur des problèmes que pose la surveillance médicale des travailleurs professionnellement exposés aux rayonnements ionisants dans les différents secteurs d'activité;

- montrer aux autorités l'importance de l'utilisation des rayonnements ionisants dans notre pays, avec ses implications spécifiques de réglementation, de contrôle et de prise en charge des accidents;

- faire bénéficier les industriels, qui s'intéressent de plus en plus aux marchés des pays en voie de développement, d'une évaluation, la plus exacte possible, de la situation actuelle en Tunisie.

* Centre national de radioprotection et Service de radiologie de l'hôpital d'enfants, place Bab Saâdoun, Tunis Jebbari, 1007 Tunis, Tunisie. 
C'est pour l'ensemble de ces raisons qu'il nous a semblé utile de présenter un bilan, le plus complet possible, de ce secteur d'activité dans notre pays incluant l'ensemble des activités de radiographie $X$ et gamma, de cristallographie et d'utilisation de sources de rayonnements diverses dans les domaines industriel et de recherche.

A notre connaissance, aucun travail similaire n'a été effectué jusqu'à ce jour en Tunisie. Notre analyse représente la photographie de la situation en Tunisie à la fin de l'année 1988, elle devra toujours être placée dans son contexte. En effet, l'évolution des infrastructures et du nombre de personnes impliquées dans l'utilisation non médicale des rayonnements ionisants est actuellement très rapide et les études proposées devront être remises à jour de manière régulière pour être exploitables.

II nous est difficile d'affirmer avec certitude que notre enquête a permis une évaluation complète et exacte de la situation, car les utilisateurs sont nombreux, dispersés dans le pays et pas toujours bien informés de la réglementation en vigueur. Cependant, nous pensons que la situation décrite correspond à la réalité avec une incertitude inférieure à $10 \%$.

\section{MODALITÉS DE L'ENQUÊTE}

Les chiffres proposés ici sont le résultat des enquêtes réalisées par le Centre national de radioprotection depuis sa création en 1971 et des déclarations établies par les utilisateurs conformément à la loi du 18 juin 1981 et à son décret d'application du 28 mars 1986 [1, 4, 5]. Les autres éléments de ce travail proviennent des différents départements du Ministère de la santé publique, de la Caisse nationale de sécurité sociale, de l'Institut national des statistiques, des archives du Conseil de l'ordre des médecins et de la pharmacie centrale de Tunisie [7-10]. En partant de l'ensemble de ces données, plusieurs tableaux et cartes géographiques sont proposés, à l'image de ce qui a été déjà décrit dans la littérature [11].

\section{RÉSULTATS (Fig. 1 à 3 )}

Les appareils utilisés en dehors du domaine médical ont été séparés en plusieurs types selon leur utilisation et le type de sources de rayonnements utilisées. C'est ainsi, que plusieurs types d'appareils ont été distingués:

- bombe au cobalt d'activité initiale de $4000 \mathrm{Ci}$ utilisée pour la stérilisation des mouches au Ministère de l'agriculture; 200 kV;

- appareils de radiographie utilisant des générateurs à rayons $X$ de 30 à

- appareils de gammagraphie utilisant des sources de cobalt 60 , de césium 137 et d'iridium 192; 


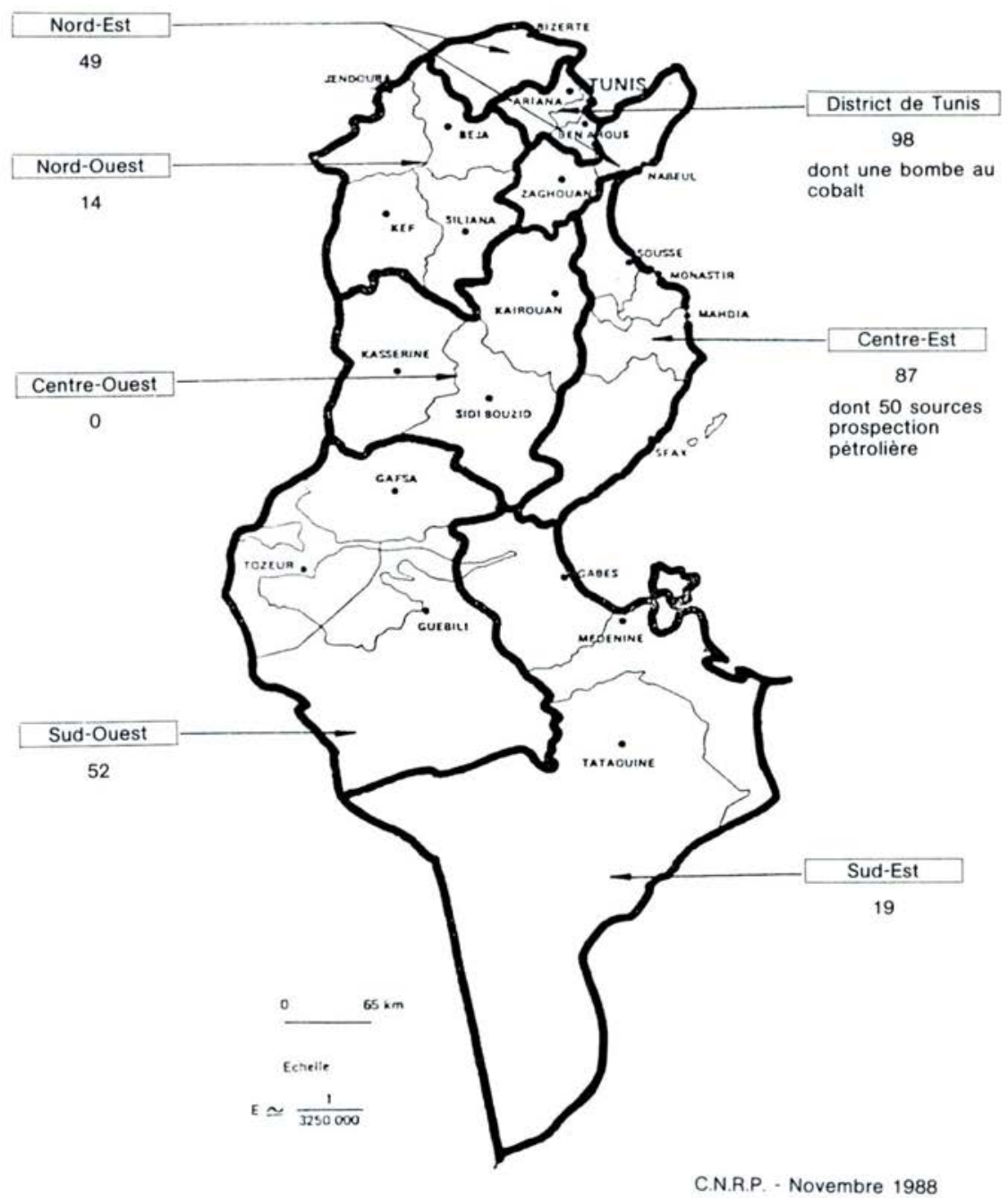

Fig. 1. - Répartition par région des sources de rayonnements ionisants (générateurs électriques de rayonnements + sources radioactives) scellées.

- appareils de mesure de densité basés sur la diffusion ou l'absorption des rayons $\gamma$ du césium 137 en général;

- appareils de mesure de la teneur en eau par détection après ralentissement des neutrons rapides émis par une source neutronique, généralement de l'américium-béryllium; 
- appareils mixtes servant à la mesure de la densité et de la teneur en eau;

- jauges de niveau fonctionnant en tout ou rien, utilisant en général des sources de césium 137 de quelques dizaines de $\mathrm{mCi}$ d'activité;

- sources radioactives scellées utilisées en prospection pétrolière pour l'analyse de la nature des sols;

- laboratoires de recherche du Ministère de l'enseignement supérieur utilisant des sources radioactives non scellées, principalement du carbone 14 , de l'iode 125 et 129 , etc...

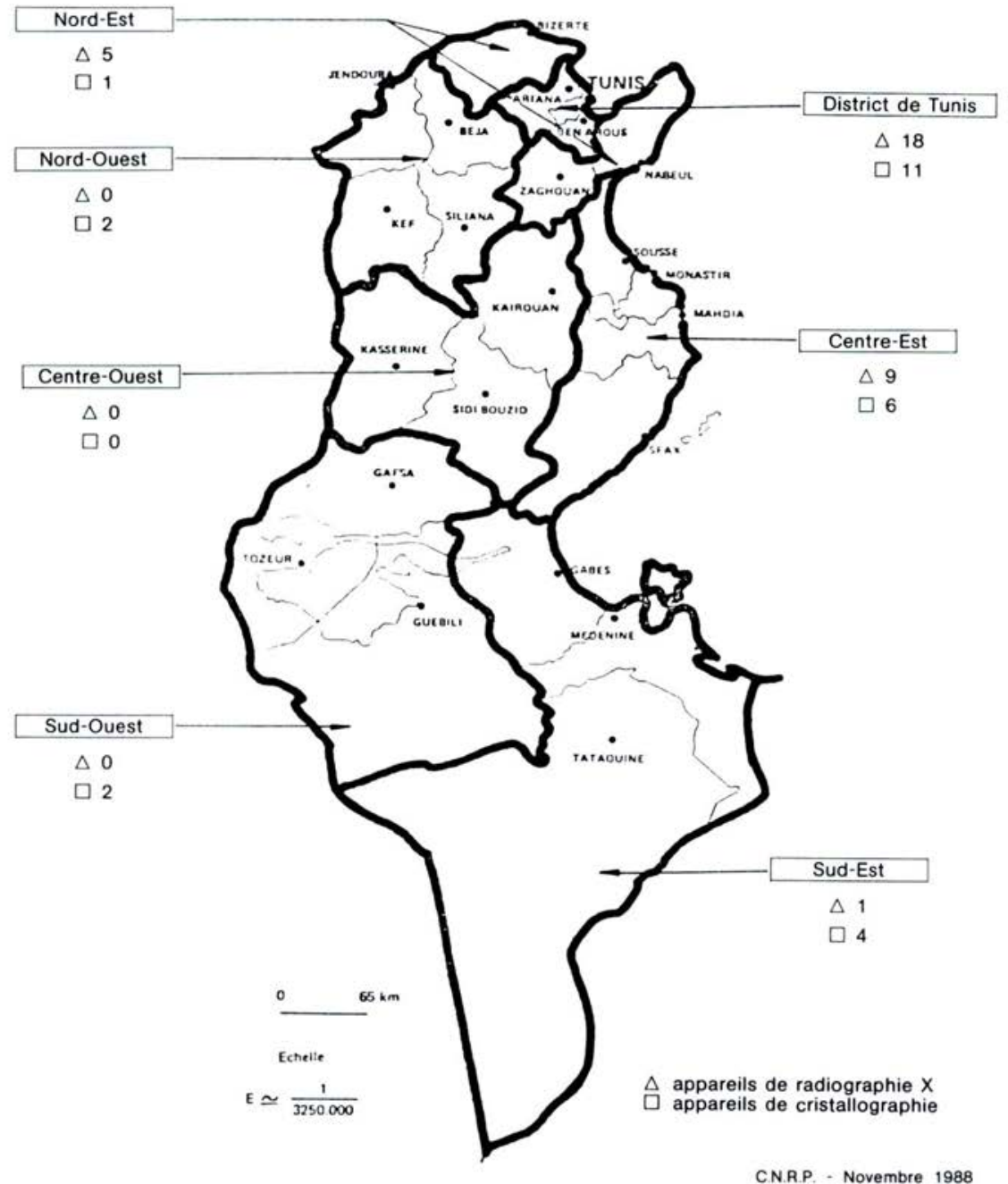

Fig. 2. - Répartition par région des générateurs électriques de rayons $X$. 


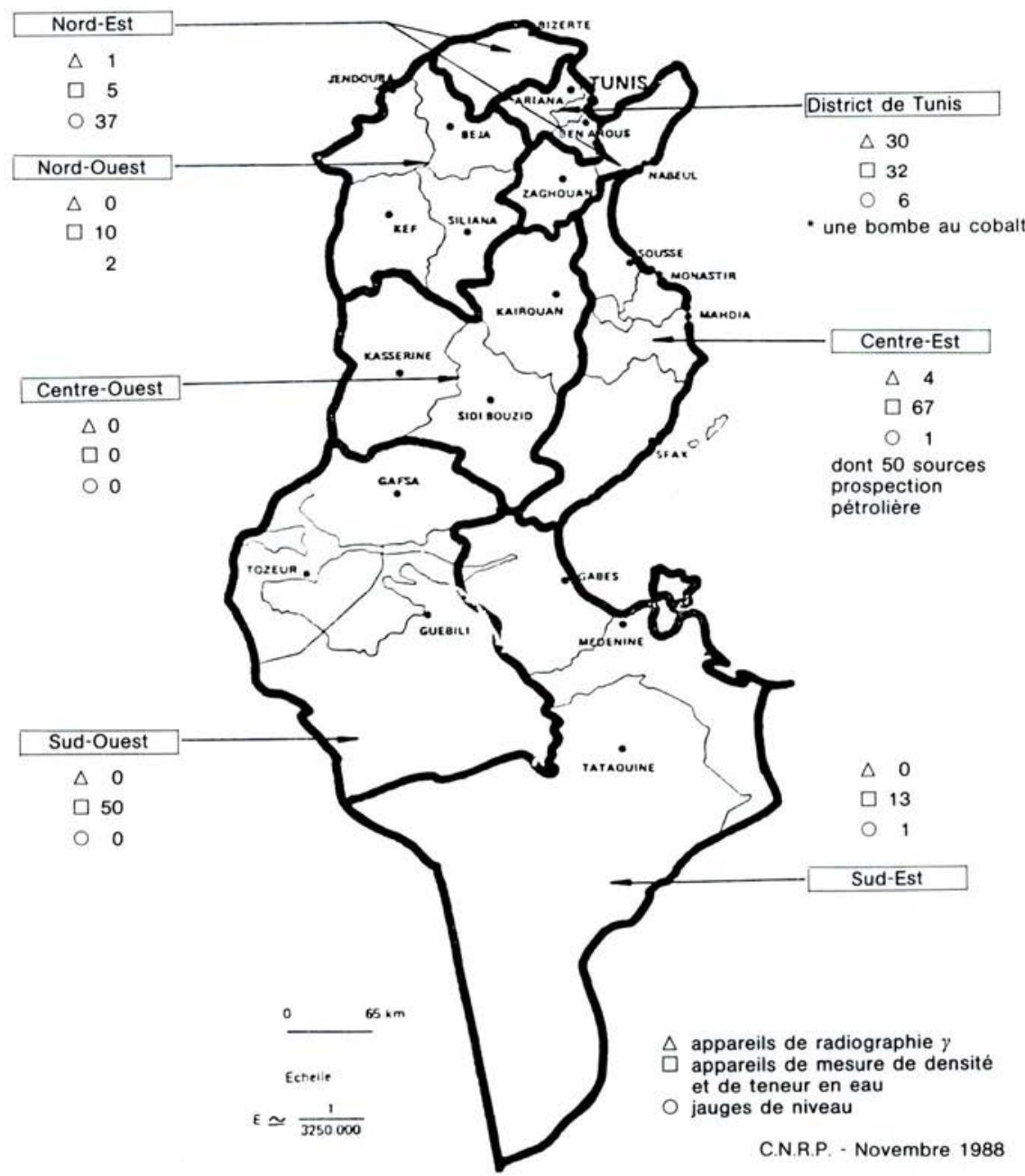

Fig. 3. - Répartition par région des appareils équipés de sources radioactives scellées.

Ces différents appareils ont été classés pour chacun des gouvernorats (préfectures) regroupés en régions selon la classification de l'Institut national tunisien des statistiques.

II est à noter que sur les 2500 travailleurs professionnellement exposés en Tunisie (dont 2000 environ sont abonnés au service de dosimétrie du Centre national de radioprotection tunisien) 400 travaillent dans le domaine non médical, dans 80 entreprises publiques et privées. La consommation de films radiographiques, l'absence d'unification des caisses de remboursement, la diversité des systèmes d'assurance sociale, la multiplication 
du nombre d'importateurs de films radiographiques, ne permettent pas d'évaluer de manière précise la consommation radiographique en Tunisie en général et la consommation radiographique industrielle en particulier. Nous avons pu évaluer la consommation des films radiographiques environ à $7 \%$ de la consommation des films à usages médicaux (tableau I) [3, 6].

TABLEAU ।

Evolution de la consommation de films radiologiques

\begin{tabular}{|c|c|c|c|}
\hline Année & $\begin{array}{c}\text { Consommation } \\
\text { en } \mathrm{m}^{2}\end{array}$ & Population & $\begin{array}{c}\text { Indice de } \\
\text { consommation } \\
\mathrm{m}^{2} / \mathrm{Hb}\end{array}$ \\
\hline 1976 & 111478 & 5727915 & $1,94.10^{-2}$ \\
\hline 1979 & 170272 & 6219800 & $2,74.10^{-2}$ \\
\hline 1982 & 247535 & 6727000 & $3,68.10^{-2}$ \\
\hline 1985 & 279657 & 7138238 & $3,92.10^{-2}$ \\
\hline
\end{tabular}

\section{DISCUSSION}

L'analyse des résultats de l'ensemble des enquêtes que nous avons effectuées amène un certain nombre de remarques importantes concernant le nombre des utilisateurs, leur diversité et les problèmes posés. En Tunisie, le nombre d'entreprises utilisant des rayonnements ionisants à but non médical est très important, avec un nombre élevé de sources puissantes utilisées dans les domaines de la gammagraphie et de la prospection pétrolière. Mais les chiffres obtenus sont largement inférieurs à ceux de la France, par exemple, où ces utilisations, en dehors des centrales nucléaires sont très répandues $[11]$.

Les cartes (fig. 1 à 3 ) montrent que ces équipements sont éparpillés dans tout le pays avec une nette prédominance au niveau des régions côtières et des régions du Sud-Est et du Sud-Ouest, où se trouvent concentrés les sites industriels, la prospection pétrolière et les mines de phosphate. Les hommes qui manipulent ces sources ont une formation variable. Certains ont obtenu leur qualification dans le cadre du programme de certification du COTEND [2] établi par le Ministère de l'économie nationale (Cétime), en collaboration avec un grand nombre d'autres ministères et le Centre national de radioprotection. Pour d'autres utilisateurs, la formation s'effectue à l'étranger ou au cours de séminaires de formation organisés par le Centre national de radioprotection sur les lieux de travail, pour les problèmes de radioprotection spécifiques. 


\section{Protection de la population, prévision des accidents}

Sachant que les sources à usage industriel sont les plus dangereuses et que ce sont elles qui sont les plus souvent incriminées dans les accidents radiologiques à travers le monde, en particulier en cas de vol, de perte etc., il a été créé une Commission nationale de radioprotection présidée par le ministre de la santé publique chargée de promouvoir la radioprotection et de préparer un plan de prévoyance et d'urgence en cas d'accidents radiologiques. Ce plan est en cours d'adoption et permettra, nous le souhaitons vivement, d'éviter à notre pays les accidents qui risquent toujours de survenir dans ce domaine d'utilisation très particulier et très dangereux du fait de l'utilisation de sources de rayonnements mobiles et transportables d'un chantier à l'autre.

\section{PERSPECTIVES D'AVENIR}

L'industrialisation de la Tunisie est en évolution rapide. L'installation de nouvelles entreprises, de nouvelles usines s'accompagne souvent d'utilisation de sources de rayonnements ionisants. Nous n'avons eu, à ce jour et fort heureusement, aucun accident majeur à déplorer du fait de l'utilisation de ces sources. II est cependant important de continuer l'effort de développement de la radioprotection fait depuis bientôt 20 ans par le Ministère de la santé publique à travers le Centre national de radioprotection afin d'éviter la survenue de tels accidents.

La meilleure façon de préparer l'avenir nous semble se résumer en les points suivants:

1. Améliorer le contrôle de l'importation, du stockage et de l'utilisation de toutes les sources de rayonnements ionisants en Tunisie, ceci permettra au Centre national de radioprotection de détenir un fichier à jour de ces sources et d'assurer le contrôle de l'utilisation saine et bénéfique de ces équipements;

2. La législation: la promulgation de textes réglementant de façon précise l'utilisation et, en particulier, le transport des sources de rayonnements ionisants, permettra de donner un outil réglementaire aux autorités compétentes pour assurer le respect des normes de radioprotection et, par conséquent, sauvegarder la santé des travailleurs professionnellement exposés et de la population en général;

3. La formation: il est nécessaire de former les utilisateurs dans le domaine de l'utilisation des rayonnements ionisants en général et en radioprotection en particulier.

Des efforts sont faits et méritent d'être étendus. La Tunisie, malgré ses faibles moyens, s'est dotée d'une structure administrative et technique qui, depuis 1971, a développé la réglementation et le contrôle de l'utilisation des rayonnements ionisants. Elle s'est dotée d'un système législatif d'avantgarde, en collaboration avec l'Agence internationale de l'énergie atomique, 
l'Organisation mondiale de la santé, le Service central français de protection contre les rayonnements ionisants et le Commissariat à l'énergie atomique français. Le contrôle rigoureux et effectif de toutes les installations utilisant des rayonnements ionisants à but médical ou non médical permettra, pour les années à venir, de faire profiter les entreprises des avantages de l'utilisation des sources de rayonnements, tout en garantissant une protection efficace de la population et des travailleurs professionnellement exposés.

\section{BIBLIOGRAPHIE}

[1] BEN SALAH M. - Evaluation du parc radiologique industriel - CES de médecine du travail, Faculté de médecine de Tunis, 1985.

[2] COMMISSION NATIONALE POUR LES CONTRÔLES NON DESTRUCTIFS. - Procédures de certification. Kassar Saïd: Secrétariat national technique (CETIME).

[3] DUPONT DE NEMOURS. - Archives, Frankfurt, RFA.

[4] GHARBI H.A. - Rapport sur l'état actuel de la radioprotection en Tunisie, perspectives d'avenir. Tunisie médicale, 1971, 41.

[5] GHARBI H.A. - Si la radiologie m'était contée. Tunisie médicale, 1984, 2.

[6] GHARBI H.A., VICEL J.C., ABDESSALEM H., DAOUES A., NAÏJA K., MTIMET S. La radiologie en Tunisie, bilan actuel, perspective d'avenir. J. Radiol., 1986, 67, 839-843.

[7] TUNISIE. INSTITUT NATIONAL DES STATISTIQUES. - Recensement général de la population et de l'habitat. 30 mars 1984 et 8 mars 1985, Vol. 1.

[8] TUNISIE. Ministère de la santé publique. - Archives de la situation administrative, février 1986.

[9] TUNISIE. Ministère de la santé publique. - Trente ans au service de la santé, 1956-1986.

[10] PHARMACIE CENTRALE DE TUNISIE. - Archives 1976-1985.

[11] SERVICE CENTRAL DE PROTECTION CONTRE LES RAYONNEMENTS IONISANTS (SCPRI). - Archives réf. $\mathrm{n}^{\circ} 11,15,16,22,25,26$. Rapports d'activité 1984-1985. 